\title{
Multi-Channel Muscle Armband Implementation: Electronic Circuit Validation and Considerations towards Medical Device Regulation Assessment
}

\author{
Martha Rocio Gonzales Loli ${ }^{1}$, Elsa Regina Vigo Ayasta ${ }^{2}$, Leyla Agueda Cavero Soto ${ }^{3}$, Jose Albites-Sanabria ${ }^{4}$ \\ Universidad Nacional Federico Villarreal, Lima, Peru ${ }^{1,2,3}$ \\ Universidad Cientifica del Sur, Lima, Peru ${ }^{4}$
}

\begin{abstract}
Multi-channel muscle arrays are commonly used as sensors in bionic prosthetic devices offering an innovative solution to recover motion in transradial amputees. This study presents preliminary assessments towards validation of a muscle armband for usage in transradial users. Analog and digital components were designed based on medical agencies' recommendations to assess future compliance with Latin American medical device regulations. The study follows two approaches, an exploratory and pre-experimental design. Design was validated and confronted among research literature and medical device regulations. For validation, a pre-experimental design was guided by a quantitative paradigm. Muscle signal was assessed before and after the condition circuit for up to four muscle signals in real time. The present study considers both the conditioning muscle signal circuit and the embedded logic implementation to record signals from the designed muscle armband. Results show that the proposed device allows to record noninvasive signals with a frequency from $20-500 \mathrm{~Hz}$.
\end{abstract}

Keywords-Component; muscle armband; surface electromyography; medical device regulation; transradial users

\section{INTRODUCTION}

According to the US Department of Health and Human Services, 200 to 600 people lose a limb every day by accident or as consequence of a disease [1]. According to Dr. Luis Farro, in Peru, amputations by accident are most prevalent on adults and young adults with a prevalence of superior amputations [2]. Main causes of hand amputations are accidents with fireworks and occupational accidents, within which the fireworks industry manufacturing has the highest percentage.

The main population segment affected by hand amputations are laborers or informal workers who usually receive a minimum wage. This type of serious accident, such as an amputation, has an important psychological impact in patients and their family. Losing the ability to perform activities of daily living, losing their autonomy, losing their role in the society, being unable to perform his trade, plunges amputees into a great frustration and depression. The injuries in this type of amputation are quite different depending on of each case. Hence, customization is an important aspect to consider in the design, as it must be perfectly adapted to the injury user specific. Available commercial solutions must comply with international and local medical devices regulations.
Currently, the Peruvian and Latin American market for limb amputations offer different types of solutions such as mechanical, electrical, myoelectric and cosmetics devices.

Mechanical prosthesis can be manufactured locally; however, due to traditional manufacturing processes, the cost of these prostheses, although not as high as myoelectric ones, is still too much for the available budget of patients. Just the device terminal (hand) of the mechanical prosthesis costs over two thousand dollars and manufacturing the prosthetic socket to suit the type of amputation of the patient is a separate cost. These prostheses are offered for forearm amputations and wrist disarticulation. The National Rehabilitation Institute (INR) offers the manufacturing service of prosthetic sockets for the two types of amputation mentioned above and the costs are on average 300 to 500 dollars depending on your insurance program.

Nevertheless, mechanical prosthetic devices are very limited in their functionality offering as much as two or three different hand movements, e.g. open and close hands to grasp items. Companies that offer myoelectric or bionic prostheses are very limited and usually imported from European countries. These devices have high costs that make it very inaccessible for frequent amputation patients with tag prices over fifty thousand dollars. An important part of the bionic prosthetic devices are sensors placed on remain parts of the patient's body, usually muscle sensors.

Analysis of surface muscle activity or electromyographic signals (sEMG) is widely used in conditions that involve diagnosis, treatment, and rehabilitation of various motor disabilities [3].

Muscle sensors measure the electrical activity generated by contracting or relaxing one or more muscle groups. A muscle sensor contains conductive elements, also called electrodes, which capture these signals. EMG activity can be recorded both non-invasively (electrodes placed on the skin) and invasively, with electrodes implanted inside nerves or around muscle fibers.

Muscle signals can be recorded in monopolar or bipolar form. Monopolar configurations refer to the use of an electrode disposed over the muscle to be measured and a reference electrode preferably located away from the muscle or in an inert area. Bipolar configurations, in contrast, are comprised of two electrodes and a reference. Usually the 
electrodes are arranged on the muscle of interest at $1-2 \mathrm{~cm}$ each to avoid capturing interference from other muscle groups, also knowns as crosswalk. The advantage of the latter configuration lies on the reduction of common noise between both electrodes [4].

Superficial muscle signals produce voltages that vary between $0-10 \mathrm{mV}$ and contain a useful range of information between $20-500 \mathrm{~Hz}$. It is recommended that the signals obtained by the electrodes (especially dry electrodes) be arranged with an analog muscle conditioning circuit. This is because a weak signal that travels large distances is susceptible to the inherent noise through the cable in which they travel. Electrodes containing a preprocessed circuit of the captured signal encapsulated in conjunction with the electrode are known as active electrodes.

When recording muscle signals, usually more than one muscle group needs to be analyzed simultaneously. This impose a particular challenge for portable systems. Components of the embedded system must be carefully chosen; microcontrollers need to be chosen carefully to suffice the requirements usually based on low power consumption while offering high and efficient processing demands.

\section{METHODS}

The present study follows two approaches, an exploratory and pre-experimental design.

In the first phase, components and designs were validated and confronted among research literature and medical device regulations.

The pre-experimental design was guided by a quantitative paradigm. Muscle signal was assessed before and after the condition circuit for up to four muscle signals in real time. Muscle armband was based on dry electrodes in bipolar configuration following methodology developed in [5].

To validate the device. A BK Precision 4040B voltage signal generator was used to vary the input of the signal conditioning circuit. Sinusoidal signals were used with frequencies from 10 to $1000 \mathrm{~Hz}$. Selected frequencies were derived after first phase analysis and discussion.

A fined machine state algorithm guided the study protocol to record muscle signals from two non-amputee users. Users were asked to perform four different gestures. Training and test data was recorded on repeated instructions. Each set of instructions lasted at least 180 seconds. A general session took about 30 minutes, the participant could stop at any requested time.

\section{Results}

Literature of over fifty research articles was revised from Scopus and Web of Science database. Literature was complemented with European and Latin American analysis of international and local medical device regulations. Table I shows main findings for device design considerations.

The European project Surface EMG for non-invasive assessment of muscles (SENIAM) proposed recommendations towards effective surface muscle signal recordings. This study takes into account these recommendations for electrode distance and positioning [6]. In addition, several studies have applied a similar configuration as proposed in Table I with successful outcomes, numbers of sensors placed on forearm vary from 2 to 8 in different studies [7,8]. The proposed system is capable of up to 8 muscle sensors recording in real time.

An embedded system was designed cable of recording up to four muscle sensors in real time. Table II shows basic requirements of the microcontroller based on input estimation and signal and classification processing load.

The above described processor characteristics will be responsible for digital signal conversions of up to four muscle signals simultaneously, signal processing of different muscle features, and muscle decoding of different hand gestures.

The signal conditioning circuit was separated into five stages: differential amplification (instrumental), high pass filter, low pass filter (1st stage), customized amplification, low pass filter (2nd stage). Logic implementation was based on four main states: reading, training, classification, emergency stop flag.

Characteristics of the recorded signal were given by the nature of a muscle signal, which has a main spectra band between $20-500 \mathrm{~Hz}[9,10]$. In order to avoid aliasing, a $2 \mathrm{kHz}$ sampling frequency was selected.

Data was acquired through eight ADC ports of the processors and saved in an array with the following structure:

[CANAL 1];[CANAL 2];[CANAL 3] ... . [CANAL 8]; [Timestamp]

Main information was comprised of each of the muscle signals and a timestamp based on clock frequency of the processor. Fig. 1 shows how data could be read once digitized by the processor.

TABLE I. DESIGN CONSIDERATIONS

\begin{tabular}{|l|l|}
\hline Categories & Observation \\
\hline Electrode type & Dry electrodes, stainless steel 316 \\
\hline Configuration & Bipolar to address common noise \\
\hline Electrode distance & $1-2 \mathrm{~cm}$ to avoid crosswalk \\
\hline Protection & Isolation circuit to prevent reverse current \\
\hline Power & Low power consumption, high precision components - \\
\hline Number of sensors & 4 to 8 muscle sensors around the arm \\
\hline
\end{tabular}

TABLE II. MicRo-CONTROLLER DESIGN

\begin{tabular}{|l|l|}
\hline Features & Details \\
\hline ADC resolution & 12 bits \\
\hline ADC channels & 4 \\
\hline Voltage & $1.8 \mathrm{~V}$ a $3.3 \mathrm{~V}$ \\
\hline Communication & I2C or UART \\
\hline Timers & 2 timers \\
\hline Clock frequency & Over $100 \mathrm{MHz}$ \\
\hline
\end{tabular}




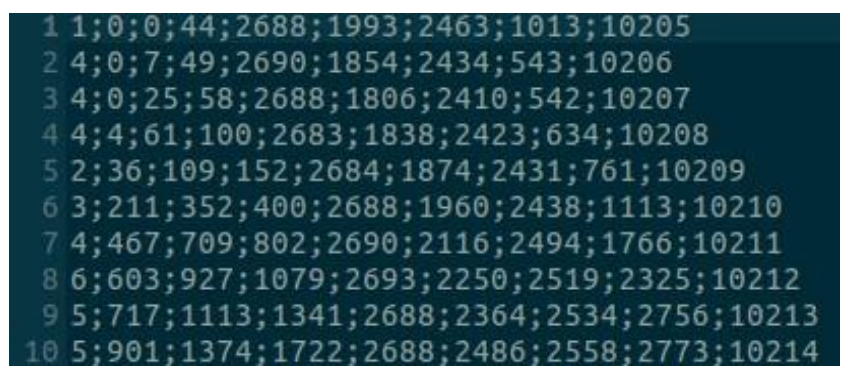

Fig. 1. EMG Data Record Test.

Several tests were performed in order to validate acquired data. In order to prevent packet lost, header and check-sum methods were implemented. Fig. 2 presents the method implementation on c-code.

UART protocol communication was chosen for data transmission. The data array showed above summed a total of 22 bytes. A sampled frequency of $2 \mathrm{KHz}$ meant information must be transferred in less than 500 milliseconds. Given those requirements, baud rate was chosen by mathematical calculation. For instance, a baud rate of 115200 Bauds, would allow a transference of 22 Bytes in $1528 \mathrm{~ms}$; this would not met the $2 \mathrm{kHz}$ sampled required frequency. In contrast 460800 Bauds, would allow packet transference in $382 \mathrm{~ms}$ which suffice system demands.

The ARM Cortex M4 offers direct memory access (DMA) for peripheral ports which offers time efficiency and does prevent the system from blocking execution while reading signals [11].

Signal classification, feature extraction and decoding algorithms were implemented in a previous study. The same study also provides information to validate the logic implementation behind the system.

Fig. 3 shows ARM Cortex M4 implementation to record fours EMG signals simultaneously from the arm of a healthy user.

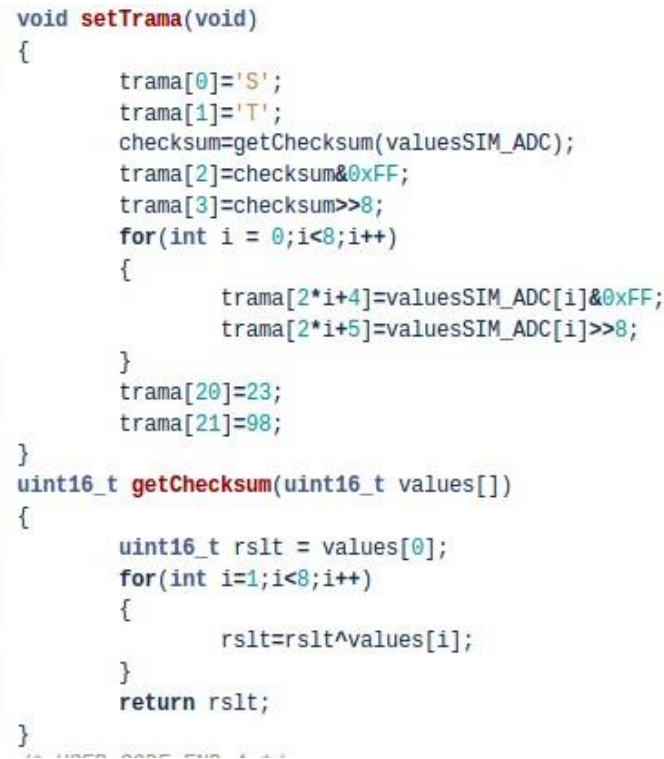

Fig. 2. C-Method Implementation.

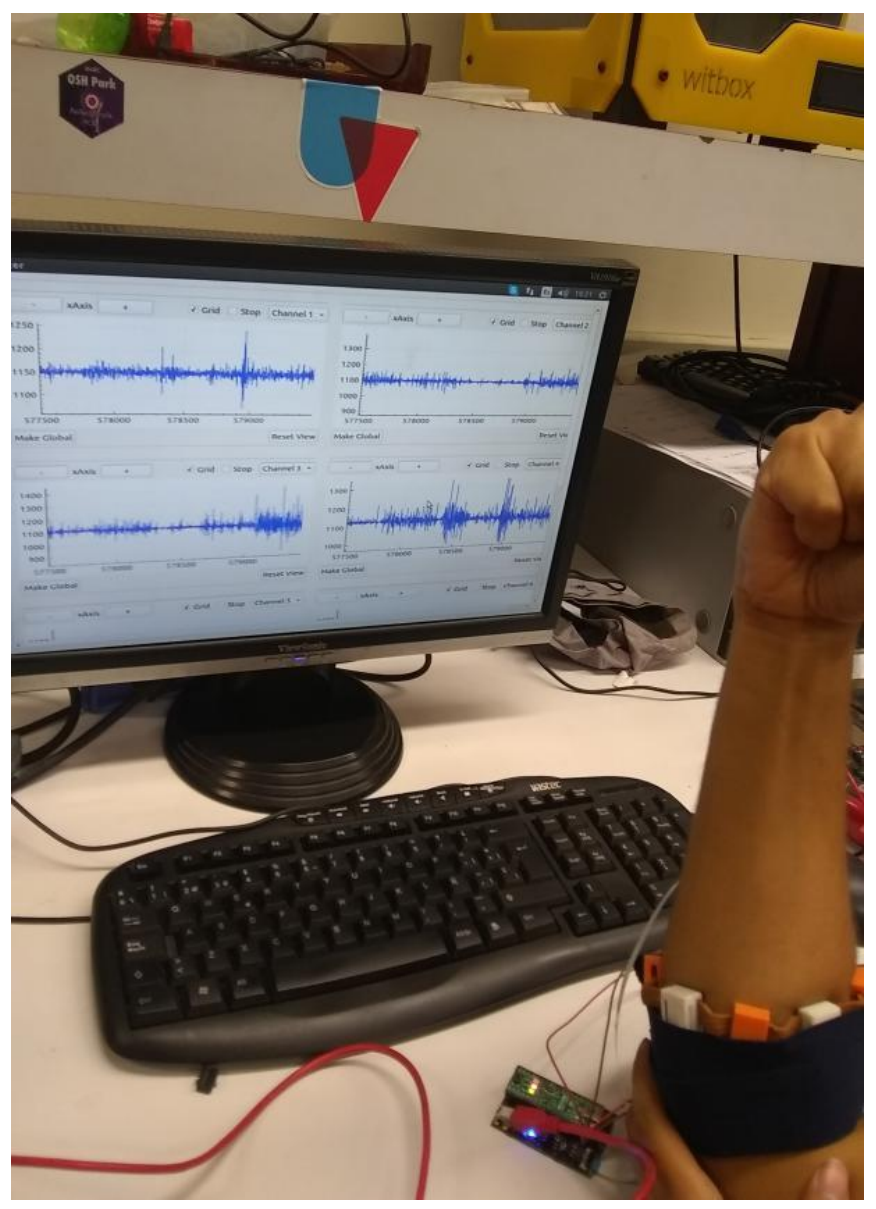

Fig. 3. Real Time Muscle Signal Recording.

Output of the instrumental amplifier and its variability with different power supplies are measured, several tests are performed controlling the offset to the circuit input.

Fig. 4 shows basic logic functionality of the system. The implemented machine state algorithms handle each of the states through several on/off flags and functions calls on each state.

After the start command, parameters and weights from the model are trained and cross validated off-line in a computer. Once the system is trained, continuous classification is performed. An emergency stop is comprised of an external input that can interrupt and stop the system at any given time.

The first test is performed using a signal with randomly chosen characteristics of $356 \mathrm{~Hz}, 6.5 \mathrm{mV}$, Senoidal, input offset: $0+-1 \mathrm{mV}$. The offset is varied in $5 \mathrm{mV}$ intervals.

It can be seen that the offset of the signal is amplified by the instrumental amplifier in proportion to its gain (11), in general offsets are suppressed largely by a high pass filter; however, this filter leaves a small offset of up to $24 \mathrm{mV}$ approximately. Which is amplified in the following stages where this offset becomes very noticeable.

Results obtained in Table III are comparable to other studies $[12,13,14]$ and thus reaffirm the potential viability of the system. 

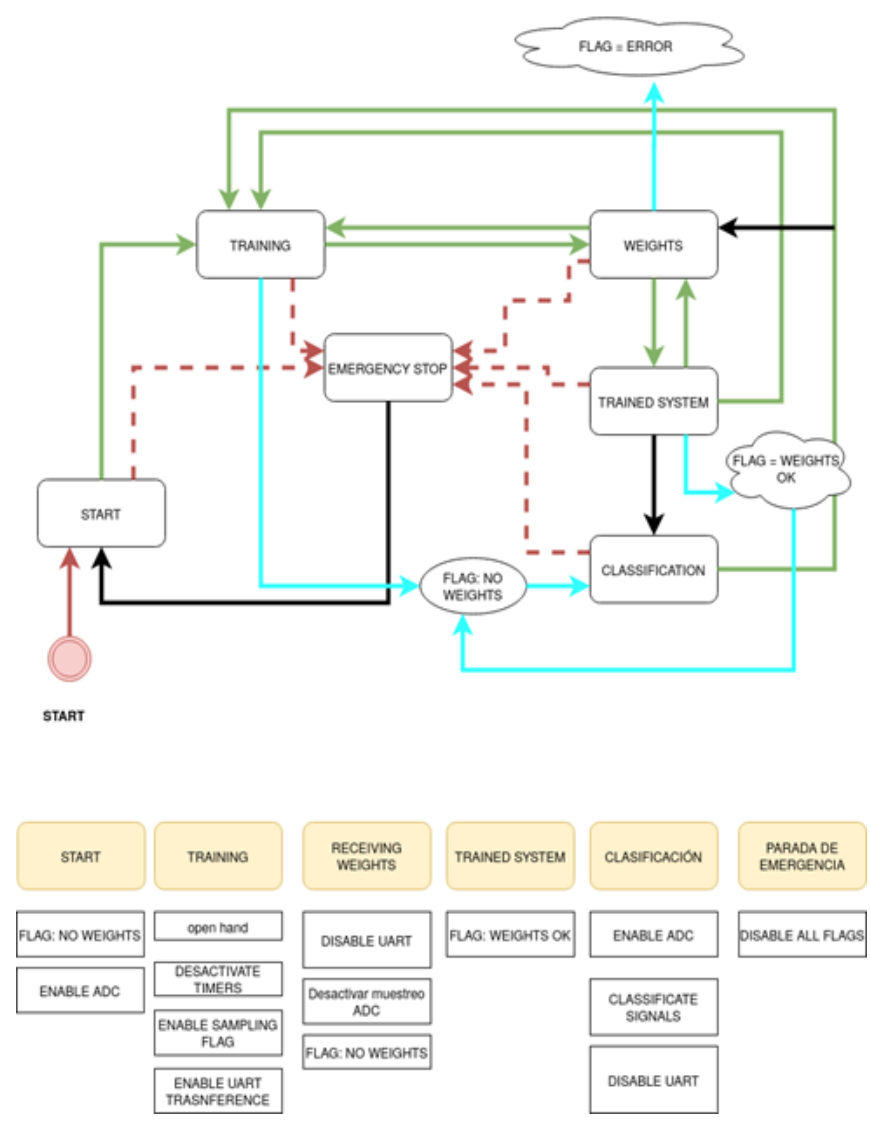

Fig. 4. Finite Machine State Implementation.

TABLE III. ANALOG TEST VALIDATION

\begin{tabular}{|l|l|l|l|l|}
\hline Input (Hz) & A & B & C & D \\
\hline $1 \mathrm{~Hz}$ & $2 \mathrm{mV}$ & $2 \mathrm{mV}$ & $20 \mathrm{mV}$ & $20 \mathrm{mV}$ \\
\hline $5 \mathrm{~Hz}$ & $7 \mathrm{mV}$ & $8 \mathrm{mV}$ & $100 \mathrm{mV}$ & $200 \mathrm{mV}$ \\
\hline $40 \mathrm{~Hz}$ & $52 \mathrm{mV}$ & $90 \mathrm{mV}$ & $3 \mathrm{~V}$ & $4.5 \mathrm{~V}$ \\
\hline $100 \mathrm{~Hz}$ & $88 \mathrm{mV}$ & $100 \mathrm{mV}$ & $4 \mathrm{~V}$ & $5 \mathrm{~V}$ \\
\hline
\end{tabular}

According to the medical and healthcare regulation authorities, muscle sensors can be classified as class I. Device system must not only comply with technical aspects such as circuit isolation and electrode medical material grade. Usage protocols and instructions are critical for device safety. Guides should include corrective and preventive actions to diminish harms in patients' health. Product development must also take into consideration some of the following regulations according to 21CFR:

- Registration: 807.

- Medical device listing: 807.

- Premarket Approval: 804.

- Investigational device exemption: 812.

- Quality system regulation: 820.

- Medical device reporting: 803 .

\section{CONCLUSIONS}

The present study considers a muscle processing circuit and the embedded logic implementation to record signals from an electromyographic sensor in compliance with local and international medical device regulations.

The present work seeks to contribute to the state of the art of EMG sensors from a technical point of view. The final goal in any sensor lies on its easiness of use and massive scale while meeting the minimum requirements for its use in both clinical and non-clinical setups.

One of the motor disabilities with the highest rate of people affected is the amputation of one of the members caused by disease or trauma [15]. The proposed solution for users who suffer some type of amputation is the replacement of the limb by an artificial limb also known as a prosthesis.

The development of prostheses in recent years has focused on improving the functionality and appearance of the artificial limb. Today, there are different prosthetic alternatives for users. From purely cosmetic alternatives, mechanical prostheses and recently bionic prostheses usually controlled by muscle or myoelectric signals. Different companies sell this type of prosthesis (BeBionics, iLimb, Touch Bionics, Vincent) with prices starting at 60 thousand dollars [16].

The field of prosthetic development is constantly growing, both at the research level and at the commercial level. Within the Peruvian Background, the biomechanics department of the National Institute of Rehabilitation develops mechanical prostheses with a cost of 7 thousand soles.

There are also initiatives that promise to reduce prosthetic costs through additive manufacturing. Within the framework of bionic prostheses, there are research projects aimed at the development of robotic prostheses in Peru by groups from different universities. Multidisciplinary and transdisciplinary skills are necessary in order to achieve results and analysis coming from specialists from different areas such as medicine, engineering, law, business among other areas [17,18]. In addition, research in these field is in compliance with sustainable development goals for 2030, especially in Peru most research is being made in sustainable water treatments [19]; however, disabilities and unemployment must be also taken into account.

However, the future work of these projects as well as the costs landed towards a commercial proposal are not fully defined until the time of writing this work. One of the important components remains the muscle sensor to be used in these prostheses, accompanied by classification algorithms that allow interpreting the movements of the person so that he can open or close the hand just by thinking about it [20].

The design of advanced and adaptive classification algorithms based on convoluted networks and non-linear filters using different signal characteristics can improve the signal [21] and its study is open to potential students interested in the use of electromyographic sensors for study and application of these in the improvement of the quality of life of people with motor disabilities. Selection and 
implementation of the processor logic and architecture must go hand-by-hand in order to meet all above described criteria.

\section{ACKNOWLEDGMENT}

The authors would like to acknowledge medical, technical, and law experts who contributed with their expertise in the different aspects of this project.

\section{REFERENCES}

[1] Ümenapf, G., \& Morbach, S., "Amputation statistics - how to interpret them?" Deutsches Ärzteblatt International, 114(8), 128, 2017.

[2] Farro, L., Tapia, R., Bautista, L., Montalvo, R., \& Iriarte, H., "Características clínicas y demográficas del paciente amputado" Revista Médica Herediana, 23(4), 240-243, 2012.

[3] Albites Sanabria, J. L., "Braingate-Enabled Intracortical Control of Commercial Tablet Computers by Individuals with Tetraplegia" Doctoral dissertation, Brown University, 2017

[4] Beck, T. W., Housh, T. J., Cramer, J. T., Malek, M. H., Mielke, M., Hendrix, R., \& Weir, J. P., "A comparison of monopolar and bipolar recording techniques for examining the patterns of responses for electromyographic amplitude and mean power frequency versus isometric torque for the vastus lateralis muscle" Journal of neuroscience methods, 166(2), 159-167, 2007

[5] Albites-Sanabria, J., "Analysis and design of an in-house low cost dry EMG sensor for bionic transradial prosthesis" International Journal of Scientific and Technology Research, 9(4), pp. 2919-2921, 2020.

[6] Stegeman, D., and H. Hermens. "Standards for surface electromyography: The European project Surface EMG for non-invasive assessment of muscles (SENIAM).", 2007.

[7] Takala, Esa-Pekka, and Risto Toivonen. "Placement of forearm surface EMG electrodes in the assessment of hand loading in manual tasks." Ergonomics, 56(7), 1159-1166, 2013

[8] Reinvee, Märt, and Mati Pääsuke. "Overview of contemporary low-cost sEMG hardware for applications in human factors and ergonomics." Proceedings of the Human Factors and Ergonomics Society Annual Meeting. Vol. 60. No. 1. Sage CA: Los Angeles, CA: SAGE Publications, 2016.

[9] Shahzad, Waseem, et al. "Enhanced Performance for Multi-Forearm Movement Decoding Using Hybrid IMU-sEMG Interface." Frontiers in neurorobotics 13, 2019.

[10] Tang, Xueyan, et al. "Hand motion classification using a multi-channel surface electromyography sensor." Sensors, 12(2), 1130-1147, 2012.
[11] Alvarado-Diaz Witman, Meneses-Claudio Brian, Fiorella FloresMedina, Patricia Condori, Natalia I. Vargas-Cuentas and Avid RomanGonzalez, "Acquisition and Classification System of EMG Signals for Interpreting the Alphabet of the Sign Language" International Journal of Advanced Computer Science and Applications(IJACSA), 10(8), 2019. http://dx.doi.org/10.14569/IJACSA.2019.0100868

[12] Heywood, Sophie, et al. "Low-cost electromyography-Validation against a commercial system using both manual and automated activation timing thresholds." Journal of Electromyography and Kinesiology, 42, 74-80, 2018.

[13] Pancholi, Sidharth, and Amit M. Joshi. "Electromyography-based hand gesture recognition system for upper limb amputees." IEEE Sensors Letters, 3(3), 1-4, 2019.

[14] Mastinu, Enzo, Max Ortiz-Catalan, and Bo Håkansson. "Analog frontends comparison in the way of a portable, low-power and low-cost EMG controller based on pattern recognition." 2015 37th Annual International Conference of the IEEE Engineering in Medicine and Biology Society (EMBC). IEEE, 2015.

[15] Shambulinga M and G. Sadashivappa, "Supervised Hyperspectral Image Classification using SVM and Linear Discriminant Analysis" International Journal of Advanced Computer Science and Applications(IJACSA), 11(10), 2020. http://dx.doi.org/10.14569/ IJACSA.2020.0111050

[16] Hofmann, M., Harris, J., Hudson, SE, \& Mankoff, J., "Helping hands: Requirements for a prototyping methodology for upper-limb prosthetics users" In Proceedings of the 2016 CHI Conference on Human Factors in Computing Systems (pp. 1769-1780). ACM, 2016.

[17] Ayala, C. E., Garro, L. L., Sanabria, F. M., Aldana, J. J., Colina, F. J., \& Albites, J. L., "Competencias interculturales en el proceso de formación en investigación en una universidad privada de Lima, Perú" Revista Espacios, 40(44), 2019.

[18] Simbron-Espejo, S. , Sanabria-Boudri, F. , Colina-Ysea, F. , AlbitesSanabria, J., "University faculty research training and performance: A case from peru" Universal Journal of Educational Research, 8(11), pp. 5053-5060, 2020.

[19] Rios-Garay, J., Cisneros-Pariona, L., Albites-Sanabria, J., “Analysis and efficiency of an affordable in-house anaerobic digester for water treatment" International Journal of Emerging Trends in Engineering Research, 8(9), pp. 6012-6016, 2020.

[20] Xu, W., Sun, Y., \& Li, C., "Research on EMG Signal Extraction Based on Biomimetic Hand" In 7th International Conference on Management, Education, Information and Control (MEICI 2017). Atlantis Press, 2017.

[21] Altin, C., \& Er, O., "Comparison of different time and frequency domain feature extraction methods on elbow gesture's EMG" European journal of interdisciplinary studies, 2 (3), 35-44, 2016. 